\title{
BIOMECHANICAL COMPARISON OF \\ LOCKED PLATING AND SPIRAL BLADE RETROGRADE NAILING OF SUPRACONDYLAR FEMUR FRACTURES
}

\author{
A Thesis \\ Submitted to \\ the Temple University Graduate Board
}

\begin{abstract}
In Partial Fulfillment
Of the Requirements for the Degree

MASTER OF SCIENCE IN MECHANICAL ENGINEERING
\end{abstract}

By
Soroush Assari
January, 2012

Thesis Approval(s):

Kurosh Darvish, Thesis Advisor, Mechanical Engineering Department

Saqib Rehman, Department of Orthopedic Surgery

Shriram Pillapakkam, Mechanical Engineering Department 


\begin{abstract}
Background: Comminuted supracondylar femur fractures in the elderly are often treated with either retrograde femoral nailing or locked plating. Early weight-bearing is typically restricted after fixing supracondylar fractures, thereby impairing the patient's mobilization. In general, surgeons are more comfortable allowing early weight-bearing of long bone fractures after nailing rather than plating, but early studies of retrograde nails for supracondylar fractures using standard distal locking showed poor fixation compared with locked plating. Newer generation distal locking techniques, such as the spiral blade, may demonstrate improved fixation, potentially allowing early weight bearing. The purpose of this study is to biomechanically compare locked plating with retrograde nailing of osteoporotic supracondylar femur fractures with simulated physiologic weight-bearing in the post-operative period.
\end{abstract}

Methods: The Locking Condylar Plate (LCP) and Retrograde/Antegrade EX Femoral Nail (RAFN) with spiral blade locking were tested using 10 paired elderly cadaveric femurs, divided into normal and low BMD groups, with a simulated AO/OTA type 33-A3 supracondylar femur fracture. Each specimen was subjected to 200,000 loading cycles simulating six weeks of postoperative recovery with full weight-bearing for an average individual and the construct subsidence and axial stiffness were measured.

Results: LCP fixation compared to RAFN showed higher axial stiffness for normal and low BMD groups (80\% and 57\% respectively). After cyclic loading, axial stiffness of both constructs decreased by $20 \%$ and RAFN fixation resulted in twice as much subsidence $(1.9 \pm 0.6 \mathrm{~mm})$. Two RAFN constructs with low BMD failed after a few cycles whereas the matched pairs fixed with LCP failed after 68,000 and 100,000 cycles. 
Conclusions: The LCP construct was stiffer than RAFN construct. Early weight bearing may cause 3-4 mm of subsidence in elderly patients with low BMD. However, because of the observed failures in two of the samples treated with RAFN in the low BMD group, early weight bearing is not recommended in osteoporotic bones treated with RAFN. 


\section{ACKNOWLEDGMENTS}

I am grateful to my advisors, Dr. Kurosh Darvish and Dr. Saqib Rehman, my wife and my friends, without whose help I would not be able to finish this work. 


\section{TABLE OF CONTENTS}

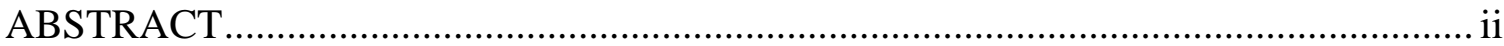

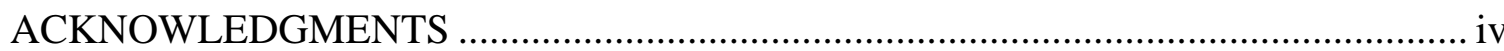

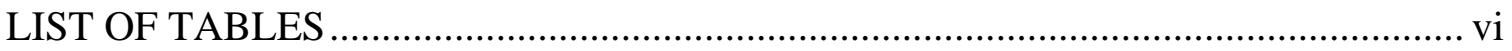

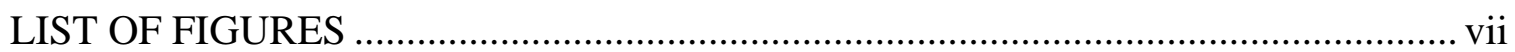

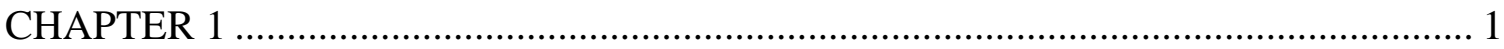

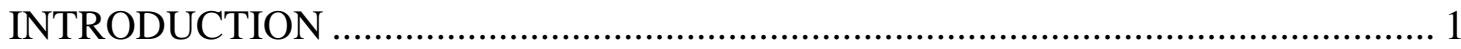

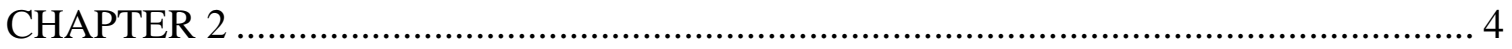

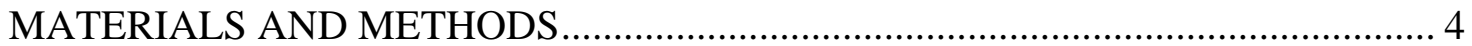

2.1 Sample Preparation ........................................................................................ 4

2.2 Experimental Setup ................................................................................... 6

2.3 Testing Procedure ........................................................................................ 7

2.4 Statistical Methods.................................................................................. 9

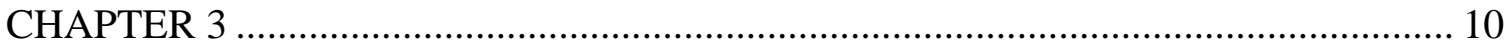

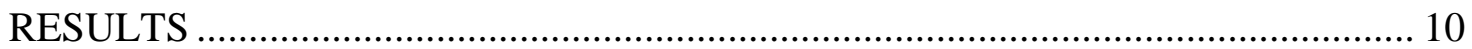

3.1 X-ray Imaging and DEXA Scan .................................................................... 10

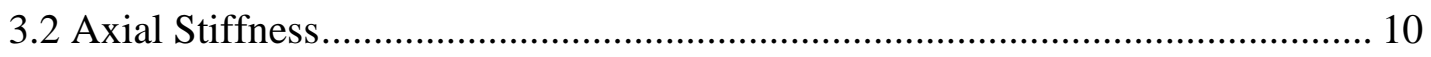

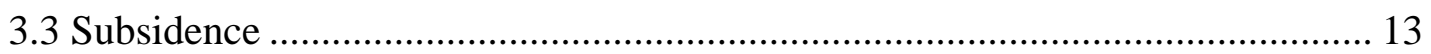

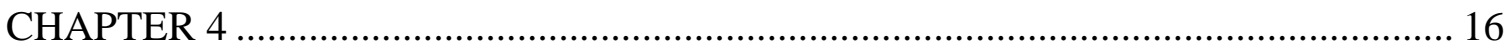

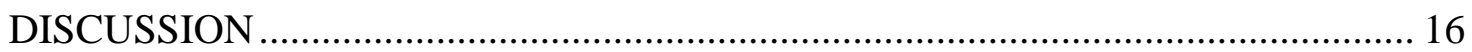

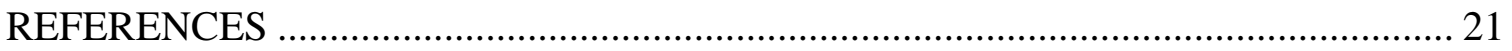




\section{LIST OF TABLES}

Table 1 - Summary of geometric measurements of cadaveric femur samples taken from

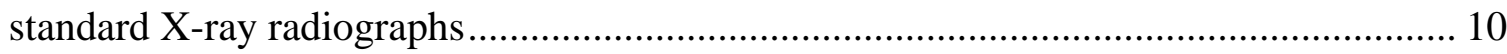

Table 2 - Summary of BMD measurements of cadaveric femur samples obtained from

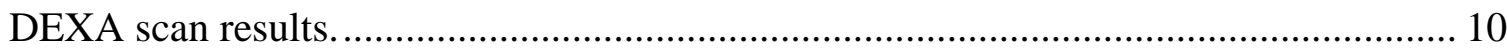




\section{LIST OF FIGURES}

Figure 1 - X-ray image of a matched pair of the RAFN (R) and LCP (L) constructs........ 5

Figure 2 - a) Experimental setup, b) Detailed view of the hip spherical joint and c) side view of the knee joint. (Legend: A: Actuator, B: Spherical Joint, C: Femur, D: Potting Material, E: Knee joint, F: Load Cell, X: Center of Rotation)

Figure $3-$ a) Typical Force-Displacement graph of a RAFN construct. Axial load is applied between $190-790 \mathrm{~N}$ and the resulting displacement is recorded. Data from two frequencies are displayed with corresponding linear fit lines. No significant difference between slopes of fit lines was observed, indicating no rate dependent effects on stiffness. b) Typical Displacement-Time graph for 50,000 cycles of axial loading corresponding to approximately 1.5 weeks of post-operative recovery of 80 minutes of ambulation per day. The distance between dotted lines is the subsidence incurred during loading. 8

Figure 4 - Stiffness of the RAFN and LCP constructs under axial compression for normal

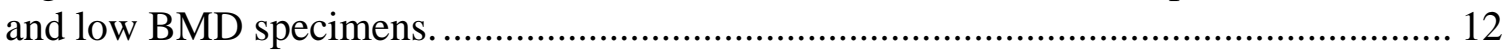

Figure 5 - Linear correlation of implant construct stiffness $\mathrm{E}$ to BMD for RAFN and LCP prior to cyclic loading. (RAFN: $E=0.25+1.09 B M D, \mathrm{R}^{2}=0.411$; $\mathrm{LCP}: E=-0.26+$

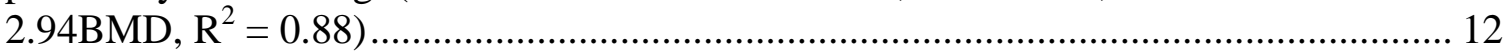

Figure 6 - RAFN and LCP Stiffness before and after cyclic loading simulating 6 weeks of post-operative recovery. 13

Figure 7 - Amount of subsidence, measured after 200,000 cycles of cyclic loading simulating 6 weeks of post-operative recovery.

Figure 8 - Linear correlation of subsidence to BMD for individual implant constructs and combined. (RAFN: $S=8.24-7.90 B M D$, LCP: $S=6.30$ - 6.14BMD, All Samples: $S=$ $6.90-6.60 B M D)$.

Figure 9 - Photographs of sample failed constructs. In RAFN constructs distal screws crushed the adjacent cortical bone and the nail protruded from the distal end of the femur ( $a$ and b). In LCP constructs failure was characterized by cracks forming at the protr.... 15 


\section{CHAPTER 1}

\section{INTRODUCTION}

Distal femoral fractures in adults are typically managed with operative methods including plating and nailing techniques. Fractures of the distal portion of the femur represent about 3.1\% of all femur fractures and occur in both young patients, as a result of high energy trauma, and elderly patients typically with osteoporosis, as a result of low energy falls.(Court-Brown and Caesar, 2006; Martinet et al., 2000) Comminution at the supracondylar region, as is seen with AO/OTA type 33-A3 fractures, has traditionally presented challenges with obtaining and maintaining adequate stabilization to allow early rehabilitation.

Before locked plating, common plating treatments of comminuted supracondylar distal femoral fractures included the fixed angle blade plate, 95 degree dynamic condylar screw and sideplate, and dual plating in order to prevent varus collapse.(Stover, 2001) Bone grafting has often been recommended as well, which has become less prevalent with the increasing use of locked lateral plates.(Bolhofner et al., 1996) Retrograde nailing methods have been available, but fixation is sometimes inadequate in these comminuted fracture types. The advent of locked plating in the distal femur has provided a very popular treatment option which has provided sufficient stabilization in these fracture patterns in osteoporotic bone. Although previous studies have shown that locked plating is biomechanically superior to standard retrograde nailing, improvements in distal locking in retrograde nails for osteoporotic bone have brought that comparison into 
question again.(WÄHNERT et al., 2010) These include features such as the spiral locked blade and multiple angled screws.

Early weight-bearing has been advocated for proximal femoral fractures in order to prevent sequelae from recumbancy and inactivity in the elderly. However, most surgeons are hesitant to allow early weight-bearing in A3 distal femoral fractures when treated with plating.(Zlowodzki et al., 2004) Conversely, many surgeons are intuitively more comfortable with allowing early weight-bearing when nailing methods are done in long bones due to the presumed "load sharing” and shorter lever arm with intramedullary nailing compared with lateral plating. Despite this sentiment, the phenomenon of loadsharing by the intramedullary implant in the widened region of the supracondylar femur is highly questionable.

The question of whether or not to use retrograde nailing or fixed angle plating for distal femoral fractures has been the focus of previous studies. However, many previous studies investigated implants which are no longer commonly used for the comminuted supracondylar femur fracture in the elderly (such as retrograde nailing with standard distal locking screws).(Higgins et al., 2007; Marti et al., 2001; Otto et al., 2009) As mentioned, relatively new fixation options are available with improved biomechanical characteristics. The Locking Condylar Plate (LCP) internal fixation system (Synthes, Paoli, PA) features combination holes for locked and unlocked plating techniques, which allows for fixed angle plating. The Retrograde/Antegrade EX Femoral Nail (RAFN) (Synthes, Paoli, PA) features a locking spiral blade option for retrograde nailing which provides additional fixation within the condylar fragments and increases the load bearing surface. Although there are few studies on these two implants, to the knowledge of the 
authors they have not been compared in one study and comparison of different studies is not possible due to biomechanical differences of their methods. Retrograde Nail with interlocked spiral blade was investigated by Ito et al. (1998 and 2001) in osteoporotic cadaveric bone with 33-A3 fracture and by Wähnert et al. (2010) in synthetic and cadaveric bone with 33-C2 type fracture. Heiney et al. (2009) utilized the same LCP and tested stiffness for 33-A type fracture and fatigue failure for 33-C type when fixed to synthetic young bones. Despite these studies, simulation of post-operative physiologic weight bearing has not been performed on fixation of 33-A3 type fracture using modern implants.

It is not well-understood whether or not lateral locked plating or intramedullary nailing with newer generation distal locking can safely allow early weight bearing in elderly patients with distal femoral fractures. Furthermore, it is unclear which method is superior in this regard. The objective of this study was to investigate and compare the biomechanical characteristics of locked condylar plating versus retrograde nailing with spiral blade locking in an elderly type A3 distal femoral supracondylar fracture model subjected to simulated postoperative weight bearing. 


\section{CHAPTER 2}

\section{MATERIALS AND METHODS}

\subsection{Sample Preparation}

10 pairs of embalmed whole femur specimens were harvested from donated cadavers aged 69 to 94 years. Standard radiographs were performed to assess for implants, bone disease, and for geometric measurements of the specimens. No specimens were found to have radiographic evidence of pathologic bone lesions. Imaging by Dual Energy X-ray Absorptiometry scan (DEXA) was performed on the specimens with a Hologic QDR 4500 (Hologic, Bedford, MA) with a fast array scan, hip protocol at the femoral neck. Specimens were placed between two bags of water to simulate thigh soft tissues. Each femur pair was assigned to one of two groups based on bone mineral density (BMD). The five pairs with a t-score less than -1 were considered as having low $\mathrm{BMD}$ and the remaining five pairs with a t-score greater than or equal to -1 were considered to have a normal BMD (Table 1).

Right sided specimens were fixed with a titanium Retrograde/Antegrade EX Femoral Nail (RAFN) with spiral blade and single screw distal interlocking and two anteroposterior proximal interlocking screws (Synthes, Paoli, PA). Pre-procedure radiographic evaluation determined that an 11x360 mm nail would fit all specimens. Left sided specimens were fixed with a stainless steel, $4.5 \mathrm{~mm}$ Locking Condylar Plate (LCP) (Synthes, Paoli, PA) with four $5.0 \mathrm{~mm}$ bicortical locking screws proximally and 5 cannulated locking screws distally (one $7.3 \mathrm{~mm}$ and four $5.0 \mathrm{~mm}$ screws). Fixation was performed according to the manufacturer's guidelines. After fixation, a $3.0 \mathrm{~cm}$ 
metaphyseal gap osteotomy was cut and bone was removed to simulate an AO/ATO type 33-A3 fracture (Figure 1). Care was taken to avoid damage to the implant.

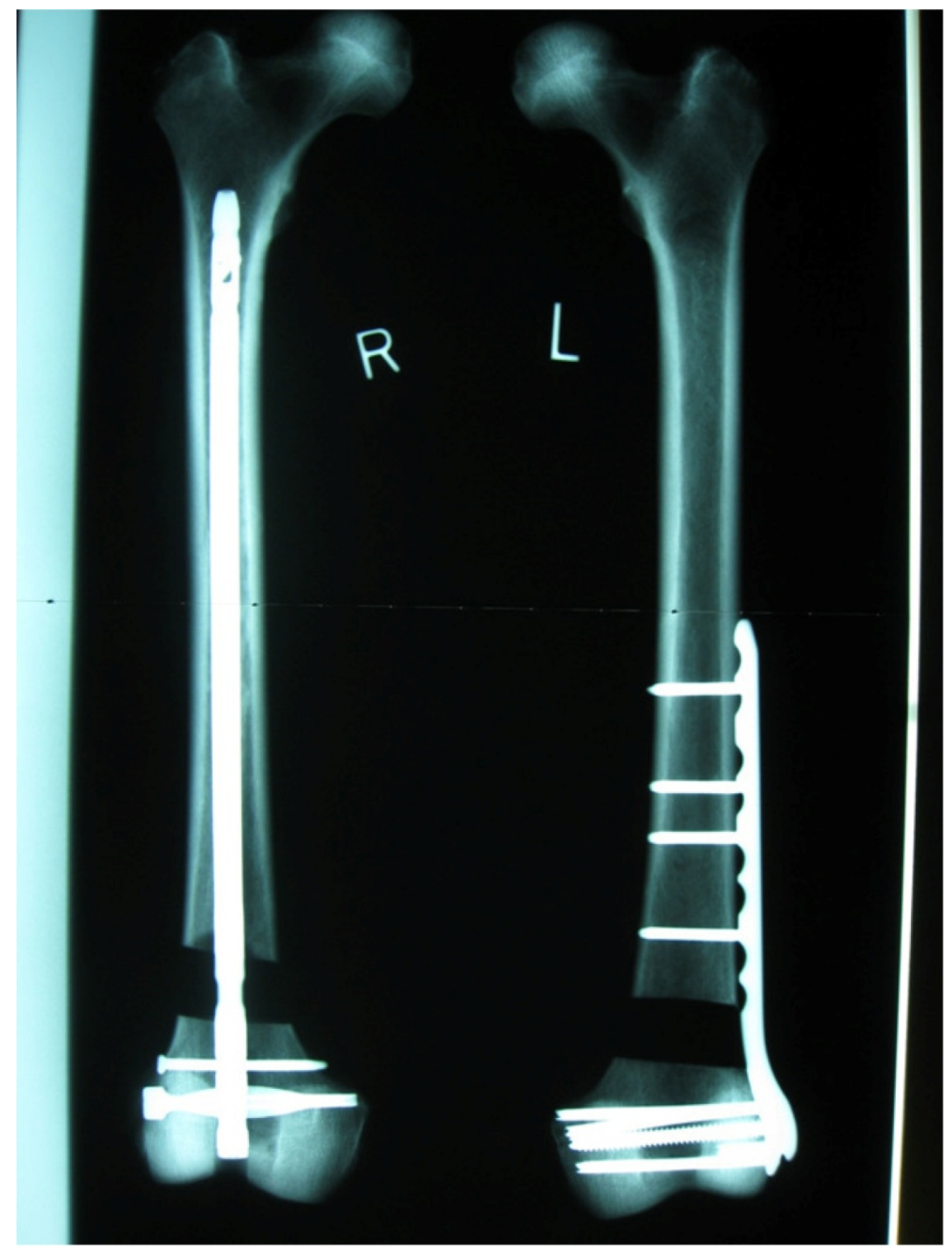

Figure 1 - X-ray image of a matched pair of the RAFN (R) and LCP (L) constructs. 


\subsection{Experimental Setup}

The experimental setup was designed to simulate normal gait with loading direction coinciding with the mechanical axis of the femur which passes through the center of the femoral head and the center of condyles (Figure 2a). The hip joint was simulated with an aluminum spherical joint with center of rotation close to the femur head center (Figure 2b). The condyles were potted under a preload of $50 \mathrm{~N}$ using Flowstone FS (Whip Mix®, Louisville, KY) in an aluminum cup allowing for free rotation in the anterior-posterior direction around the center of condyle similar to a knee joint (Figure 2c). To ensure that the potting material did not have contact with the implant and screws, foam was used to cover the implant and screws during potting, and after it cured, the foam was removed to allow observation of the screws and implant in case of failure. 

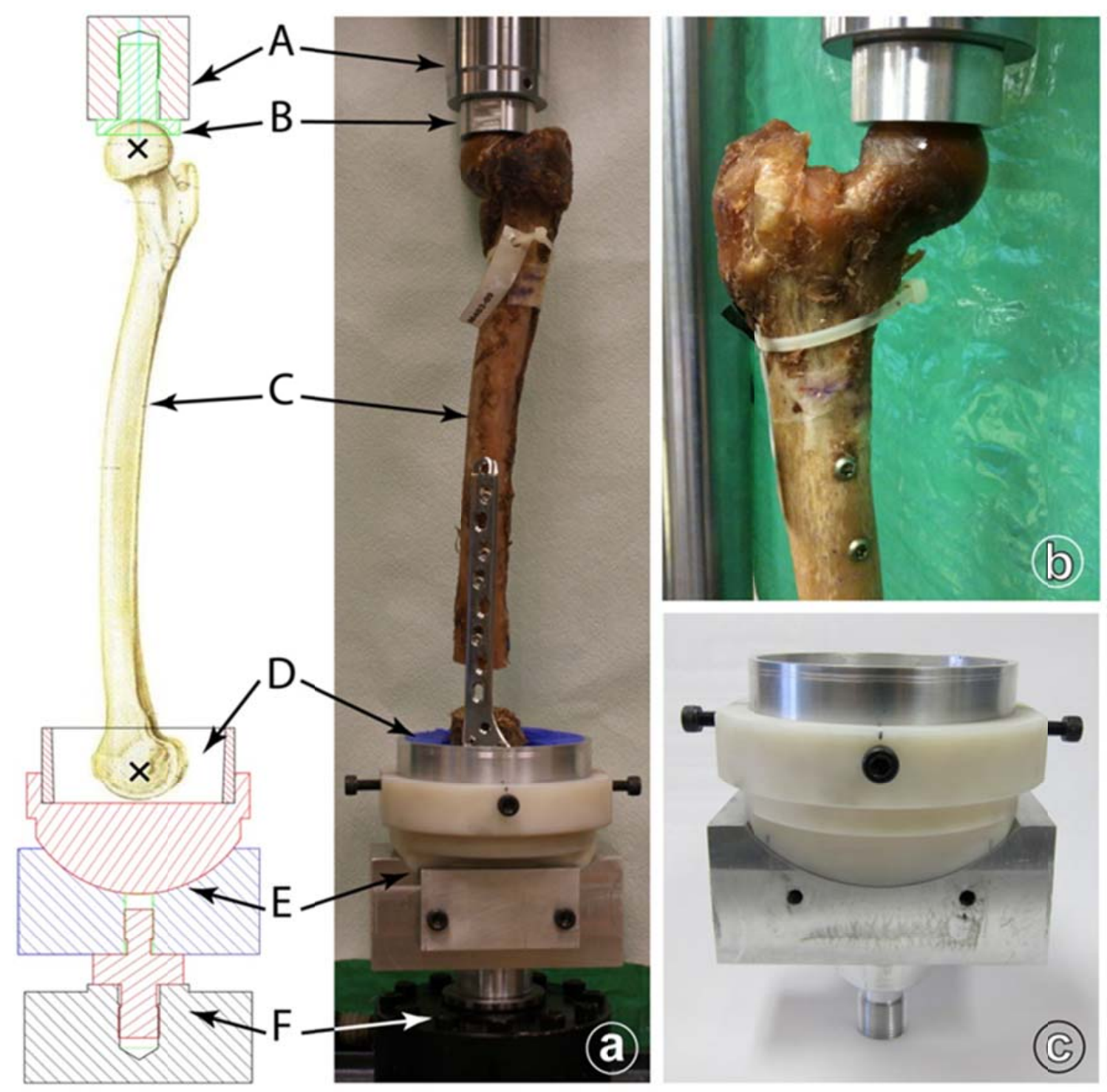

Figure 2 - a) Experimental setup, b) Detailed view of the hip spherical joint and c) side view of the knee joint. (Legend: A: Actuator, B: Spherical Joint, C: Femur, D: Potting Material, E: Knee joint, F: Load Cell, X: Center of Rotation)

\subsection{Testing Procedure}

The MTS Landmark 370.10 Test System (MTS, Eden Prairie, MN) was used to apply an axial load to each implant construct and measure the displacement. To determine the construct stiffness, each specimen was tested under a sinusoidal 
compression force with maximum and minimum of $200 \mathrm{~N}$ and $800 \mathrm{~N}$ (approximately one body weight) and frequencies of 1 and $10 \mathrm{~Hz}$. Different frequencies were tested to study the rate dependency of stiffness. In order to calculate axial stiffness of the bone-implant structure, the deformation of the two ends of the femur and the applied axial load were measured and recorded. The slope of regression line to 5 complete cycles of loading and unloading in force-displacement graph was considered as the stiffness (Figure 3a).
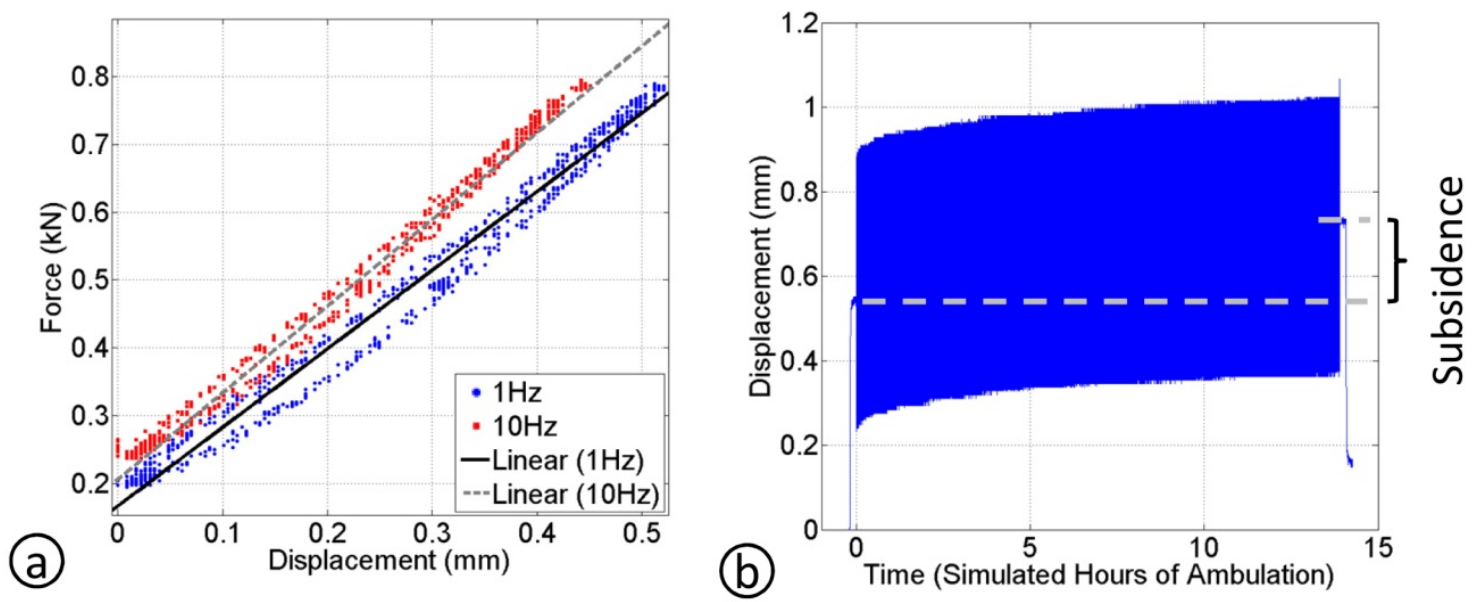

Figure 3 -a) Typical Force-Displacement graph of a RAFN construct. Axial load is applied between $190-790 \mathrm{~N}$ and the resulting displacement is recorded. Data from two frequencies are displayed with corresponding linear fit lines. No significant difference between slopes of fit lines was observed, indicating no rate dependent effects on stiffness. b) Typical Displacement-Time graph for 50,000 cycles of axial loading corresponding to approximately 1.5 weeks of post-operative recovery of 80 minutes of ambulation per day.

The distance between dotted lines is the subsidence incurred during loading.

To simulate a six-week postoperative recovery period in which patient starts with light ambulation for two hours per day, first 100,000 cycles of compression force of 190 - $790 \mathrm{~N}$ (with maximum of approximately one body weight, $75 \mathrm{~kg}$ ) were applied on each sample to simulate first three weeks and additional 100,000 cycles with higher load range 
of $290-1180 \mathrm{~N}$ (average of one body weight) were applied to simulate second three weeks in which patient puts more weight on the injured leg. The cyclic loading was applied at $10 \mathrm{~Hz}$ in order to limit the test time to two days for each specimen. Before and after each series of cyclic loading, a static load of $735 \mathrm{~N}$ (75 kg) was applied for one

minute. The difference between the positions during the static loading is defined as the subsidence incurred during the cyclic loading (Figure 3b). Stiffness of the bone-implant was measured again after the whole 200,000 cycles to investigate any significant change.

\subsection{Statistical Methods}

Statistical analysis was performed using JMP 8.0.1 software (SAS Institute Inc., Cary, NC). Normality of distributions was tested using the Kolmogorov-SmirnoffLillefors test. A matched paired t-test was used to compare the stiffness of the boneimplant structures and the subsidence with respect to the implant type and BMD groups. The relationship between stiffness and subsidence with respect to BMD was compared using linear regression analysis and F-test. In significance tests, two sided p-values less than 0.05 were considered significant. Values reported represent the mean \pm the standard error of the mean. 


\section{CHAPTER 3}

\section{RESULTS}

\subsection{X-ray Imaging and DEXA Scan}

All geometrical measurements and DEXA results for normal and low BMD groups are summarized in Tables 1 and 2. No significant difference was found between the BMD of right and left paired samples $(p=0.18)$ and the difference between BMD groups was found to be statistically significant $(p<0.0001)$.

Table 1 - Summary of geometric measurements of cadaveric femur samples taken from standard X-ray radiographs

\begin{tabular}{|c|c|c|c|c|c|c|c|}
\cline { 2 - 7 } \multicolumn{1}{c|}{} & $\begin{array}{c}\text { Femoral } \\
\text { Length } \\
\mathbf{( m m )}\end{array}$ & $\begin{array}{c}\text { Head } \\
\text { Diameter } \\
\mathbf{( m m}\end{array}$ & $\begin{array}{c}\text { Condyle } \\
\text { Width } \\
\mathbf{( m m}\end{array}$ & $\begin{array}{c}\text { Midshaft } \\
\text { Diameter } \\
\mathbf{( m m )}\end{array}$ & $\begin{array}{c}\text { Midshaft } \\
\text { Cortex } \\
\text { Thickness } \\
(\mathbf{m m})\end{array}$ & $\begin{array}{c}\text { Age } \\
\text { (years) }\end{array}$ & Gender \\
\hline $\begin{array}{c}\text { Normal } \\
\text { BMD }\end{array}$ & $475 \pm 3.0$ & $51.2 \pm 1.3$ & $51.0 \pm 0.8$ & $32.0 \pm 2.0$ & $10.4 \pm 1.2$ & $83 \pm 3.1$ & $5 \mathrm{M}$ \\
\hline Low BMD & $475 \pm 12.5$ & $47.5 \pm 1.7$ & $45.4 \pm 1.4$ & $28.2 \pm 1.5$ & $6.8 \pm 0.4$ & $77.6 \pm 3.3$ & $4 \mathrm{~F}, 1 \mathrm{M}$ \\
\hline
\end{tabular}

Table 2 - Summary of BMD measurements of cadaveric femur samples obtained from DEXA scan results.

\begin{tabular}{|c|c|c|c|c|c|c|}
\hline & \multicolumn{2}{|c|}{ BMD $\left(\mathrm{g} / \mathrm{cm}^{2}\right)$} & \multicolumn{2}{|c|}{$t$-score } & \multicolumn{2}{|c|}{$z$-score } \\
\hline & Left & Right & Left & Right & Left & Right \\
\hline Normal BMD & $0.91 \pm 0.05$ & $0.92 \pm 0.02$ & $-0.80 \pm 0.16$ & $-0.74 \pm 0.14$ & $0.25 \pm 0.17$ & $0.45 \pm 0.15$ \\
\hline Low BMD & $0.55 \pm 0.08$ & $0.49 \pm 0.08$ & $-3.26 \pm 0.66$ & $-3.52 \pm 0.62$ & $-1.30 \pm 0.76$ & $-1.7 \pm 0.72$ \\
\hline
\end{tabular}

\subsection{Axial Stiffness}

The axial stiffness was increased $7 \%$ and $10 \%$ for LCP and RAFN respectively from $1 \mathrm{~Hz}$ to $10 \mathrm{~Hz}$ cyclic loading ( $p<0.0001)$. The reported stiffness values are at $1 \mathrm{~Hz}$. 
The LCP bone-implant construct was consistently stiffer than the RAFN in axial compression. Prior to cyclic loading, the average difference between the LCP and RAFN among normal BMD samples was $1.10 \mathrm{kN} / \mathrm{mm}(p=0.005)$ and among low BMD samples the average difference was $0.47 \mathrm{kN} / \mathrm{mm}(p=0.008)$ (Figure 4$)$. The stiffness of the constructs was found to correlate linearly to the BMD (Figure 5) and were significantly different from each other $(p=0.0002)$. Statistical results showed BMD was a stronger predictive measure of stiffness, although correlation was acceptable with tscore (RAFN: $E=1.35+0.16 \mathrm{t}, \mathrm{R}^{2}=0.396$; $\mathrm{LCP}: E=2.70+0.41 \mathrm{t}, \mathrm{R}^{2}=0.861$ ) and $\mathrm{z}-$ score (RAFN: $E=1.65+0.16 \mathrm{z}, \mathrm{R}^{2}=0.306$; $\left.\mathrm{LCP}: E=2.09+0.42 \mathrm{z}, \mathrm{R}^{2}=0.804\right)$. No significant correlation between axial stiffness and geometrical bone measurements was found, including femoral length (RAFN $\mathrm{R}^{2}=0.059$, LCP $\mathrm{R}^{2}=0.066$ ), midshaft cortex thickness $\left(\right.$ RAFN $\left.\mathrm{R}^{2}=0.39, \mathrm{LCP} \mathrm{R}^{2}=0.48\right)$, femoral head diameter $\left(\mathrm{RAFN} \mathrm{R}^{2}=0.11\right.$, LCP $\left.\mathrm{R}^{2}=0.23\right)$, midshaft diameter $\left(\mathrm{RAFN} \mathrm{R}^{2}=0.0003, \mathrm{LCP}^{2}=0.037\right)$, and condyle width (RAFN R ${ }^{2}=0.0002$, LCP $\left.^{2}=0.077\right)$. Following 200,000 cycles of axial loading, an average decrease in axial stiffness of $0.39 \mathrm{kN} / \mathrm{mm}$ was observed for samples fixed with LCP ( $p=0.006)$, however, an average decrease of $0.42 \mathrm{kN} / \mathrm{mm}$ in the RAFN was not statistically significant $(p=0.13)$ (Figure 6). 


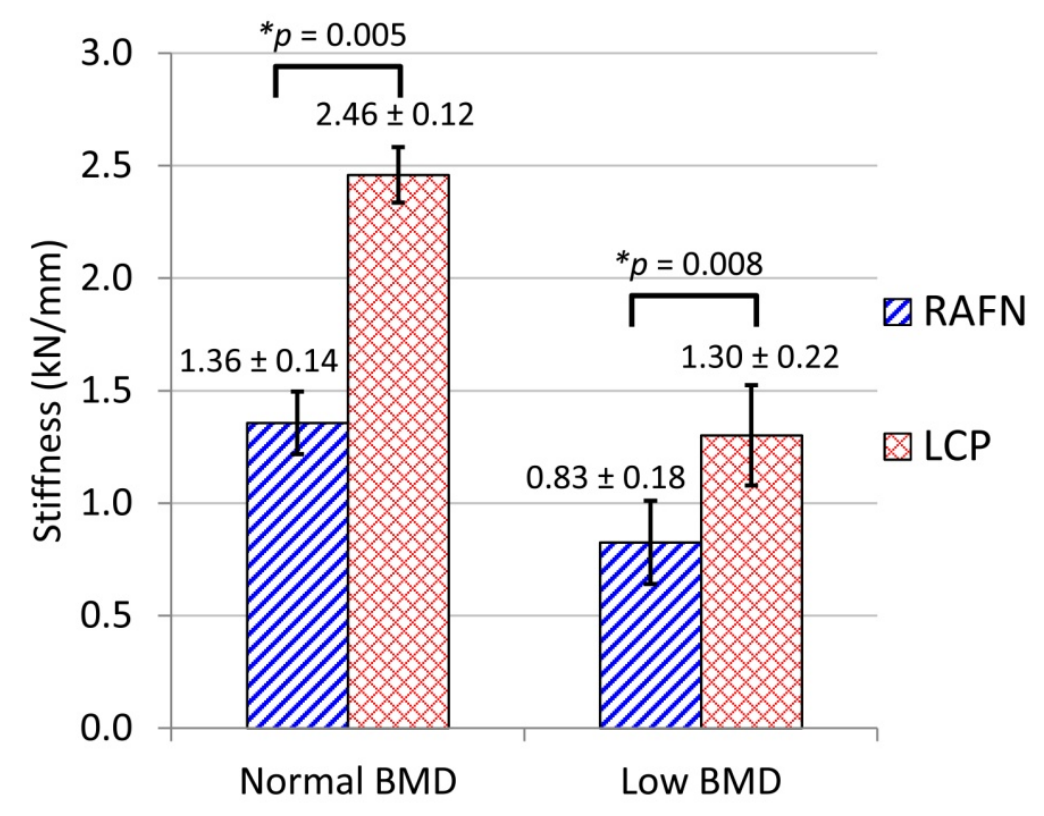

Figure 4 - Stiffness of the RAFN and LCP constructs under axial compression for normal and low BMD specimens.

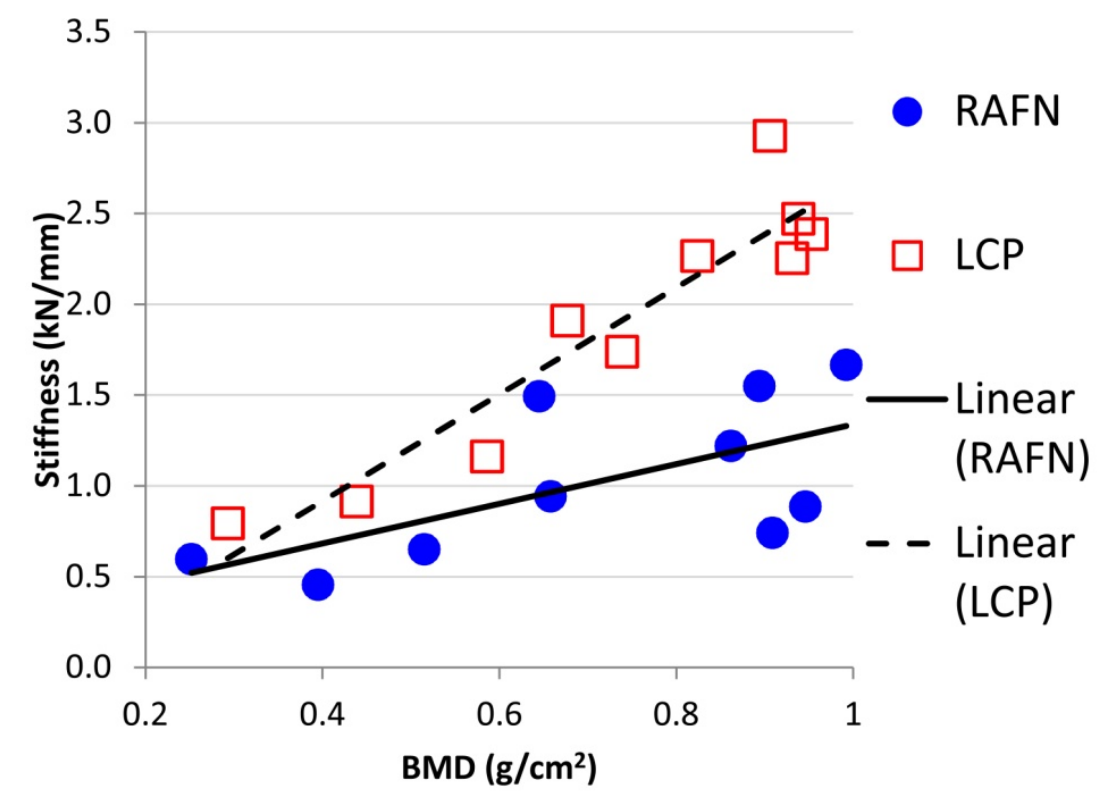

Figure 5 - Linear correlation of implant construct stiffness E to BMD for RAFN and LCP prior to cyclic loading. (RAFN: $E=0.25+1.09 B M D, \mathrm{R}^{2}=0.411 ; \mathrm{LCP}: E=-0.26+$ 2.94BMD, $\left.\mathrm{R}^{2}=0.88\right)$ 


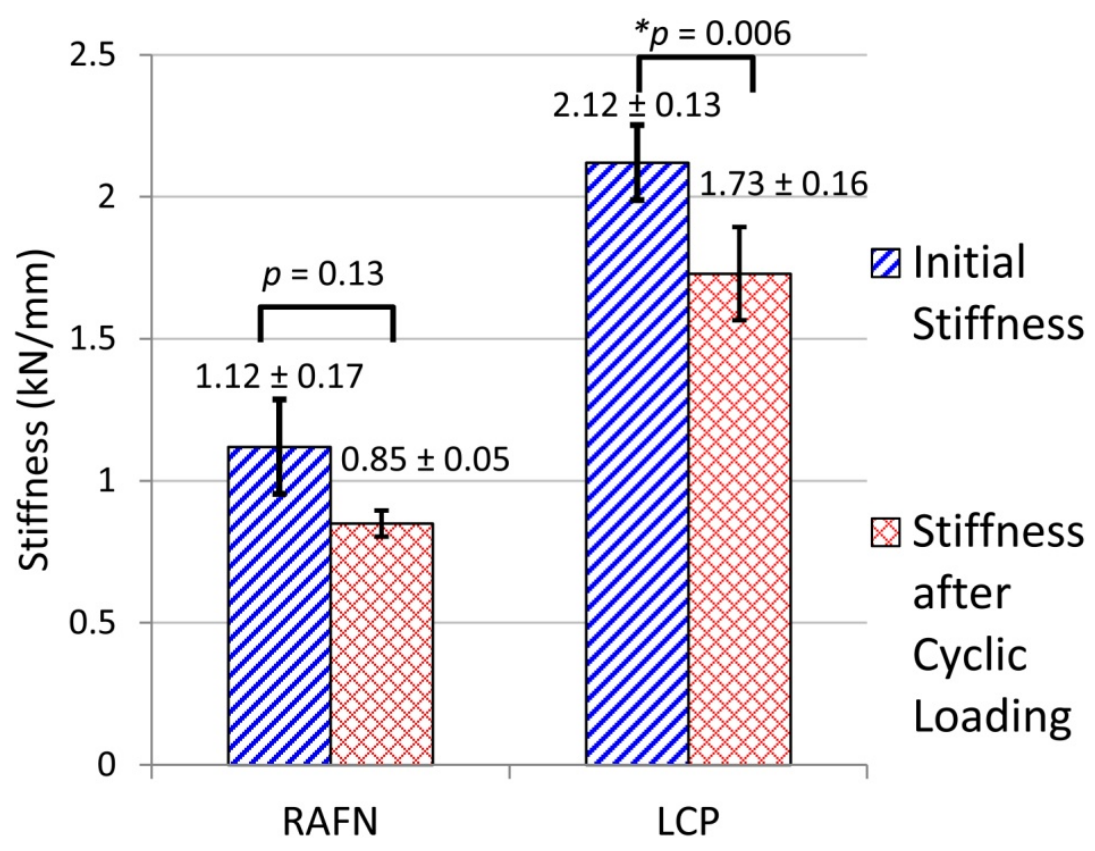

Figure 6 - RAFN and LCP Stiffness before and after cyclic loading simulating 6 weeks of post-operative recovery.

\subsection{Subsidence}

Figure 7 summarizes the measured subsidence for each BMD group. LCP constructs deformed an average of $1.0 \mathrm{~mm}$ less than RAFN $(p=0.066)$. Among normal BMD samples, the LCP deformed $0.4 \mathrm{~mm}$ less than the RAFN ( $p=0.056$ ), however, the difference between low BMD samples was not statistically significant $(p=0.18)$. BMD was found to correlate linearly to the amount of subsidence observed (Figure 8) (LCP: $\mathrm{R}^{2}$ $=0.567$, RAFN: $\left.\mathrm{R}^{2}=0.902\right)$, however the two regression lines were not statistically different $(p=0.24)$, therefore a combined regression line for all samples was created $\left(\mathrm{R}^{2}\right.$ $=0.657)$. Prior to start of cyclic loading, two RAFN constructs failed catastrophically (nail protruded into the articular surface), and the two matched pairs fixed with LCP failed during cyclic loading after 68,000 and 100,000 cycles (specimens with t scores of - 
5.7 and -3.3, respectively). Failure was defined to have a subsidence more than $6 \mathrm{~mm}$ since the implant got close to the potting material. In low BMD group subsidence was occurred mostly in distal femur for both implants as well as protrusion of the distal screw proximal to the fracture for specimens fixed with LCP (Figure 9).

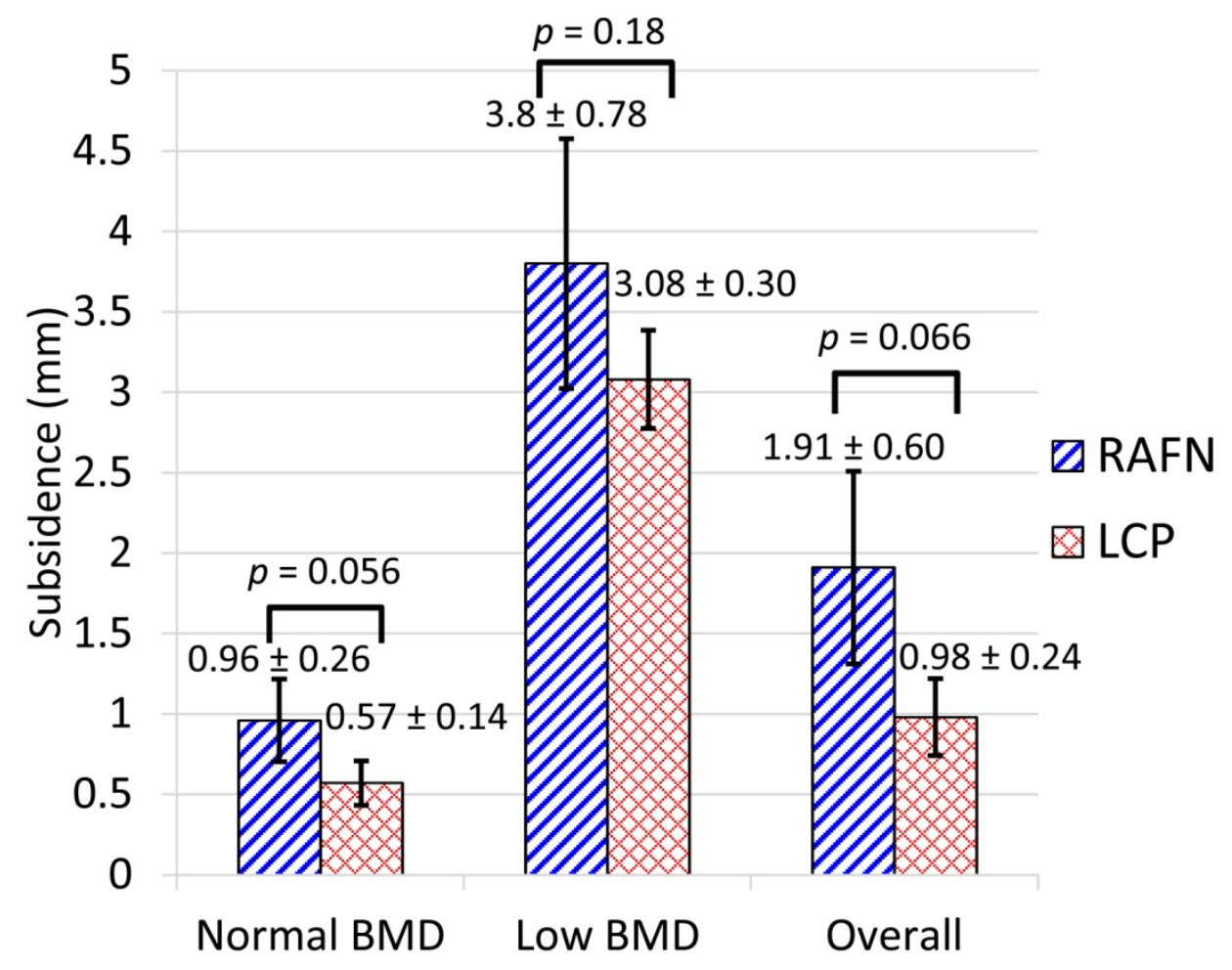

Figure 7 - Amount of subsidence, measured after 200,000 cycles of cyclic loading simulating 6 weeks of post-operative recovery. 


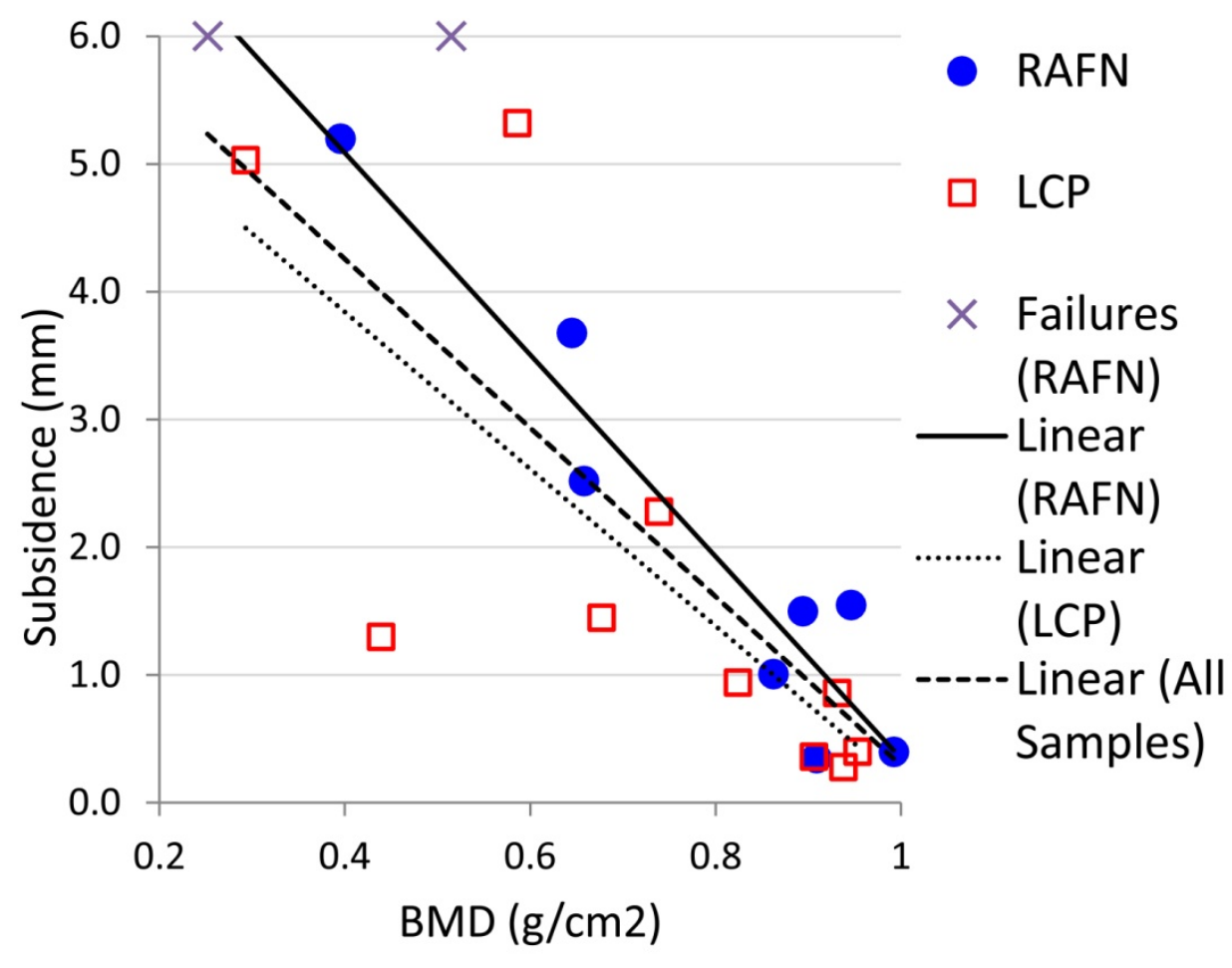

Figure 8 - Linear correlation of subsidence to BMD for individual implant constructs and combined. (RAFN: $S=8.24-7.90 B M D$, LCP: $S=6.30-6.14 B M D$, All Samples: $S=$ $6.90-6.60 B M D)$

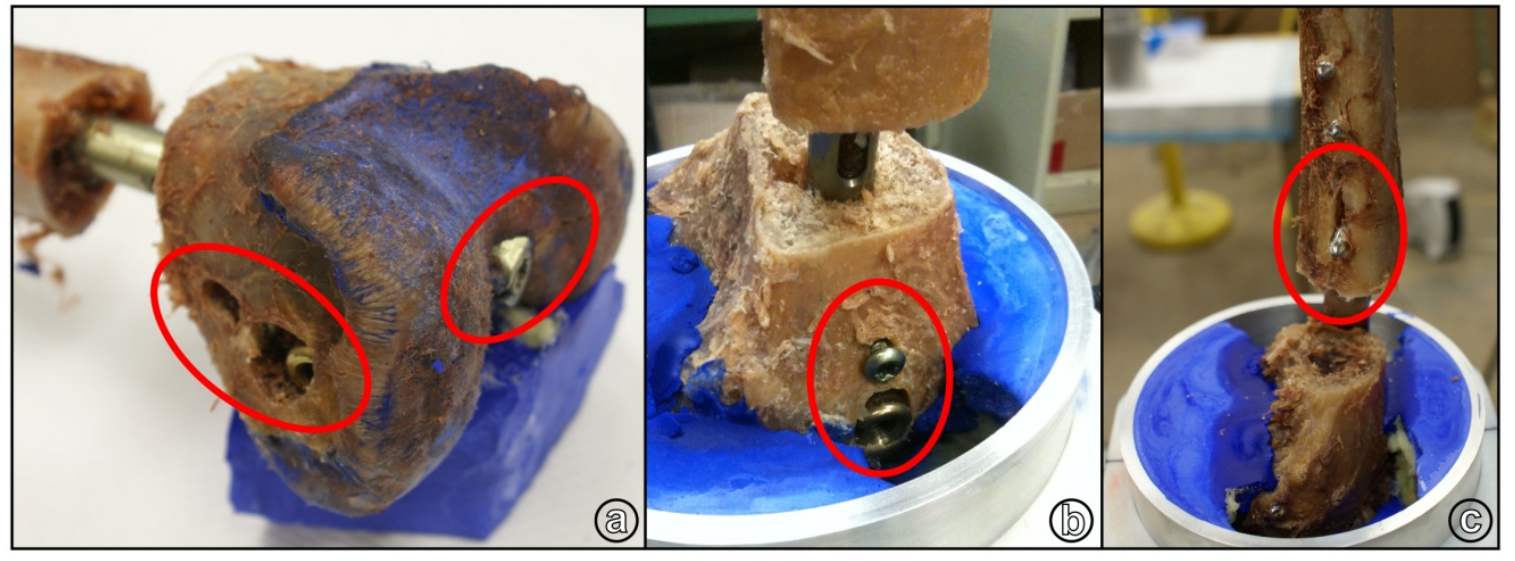

Figure 9 - Photographs of sample failed constructs. In RAFN constructs distal screws crushed the adjacent cortical bone and the nail protruded from the distal end of the femur ( $a$ and $b$ ). In LCP constructs failure was characterized by cracks forming at the protr 


\section{CHAPTER 4}

\section{DISCUSSION}

This study investigated the biomechanical differences between two common internal fixation devices, Locked Condylar Plate (LCP) and Retrograde/Antegrade EX Femoral Nail (RAFN), for osteoporotic comminuted supracondylar distal femoral fractures in elderly patients. Although other studies have been reported on each implant, this study was designed to allow a closer simulation of physiological post-operative weight-bearing after fixation using implants that are specifically designed to improve fixation in osteoporotic bone.

LCP fixation compared RAFN showed higher axial stiffness for normal and low BMD groups ( $80 \%$ and $57 \%$ respectively). This difference was more pronounced among normal BMD specimens and BMD in both cases was a strong predictor of the overall stiffness. After cyclic loading, axial stiffness of both constructs decreased by $20 \%$.

RAFN fixation resulted in twice as much subsidence ( $3.8 \pm 0.8 \mathrm{~mm}$ for low BMD and $1.9 \pm 0.6 \mathrm{~mm}$ overall), however, such difference may not be of clinical significance. Whereas this difference is less in normal bone, the results suggest that even with enhanced distal fixation, RAFN's have greater loss of fixation in osteoporotic bone. Our observations that the majority of subsidence occurs due to bone failure at the site of screws, as opposed to deformation within the screws or toggling, are consistent with previous studies.(Higgins et al., 2007; Marti et al., 2001; WÄHNERT et al., 2010) 
Results relating to the low BMD group are of particular clinical interest, as many fractures occur in patients suffering from a lower than normal BMD. It is important to note that a correlation between BMD and subsidence was demonstrated for both implants in this study, but the difference in subsidence between the two implants was less than 1 mm (3.1 mm vs. $3.8 \mathrm{~mm}$ in low BMD group). This is arguably not clinically significant despite the fact that the LCP was biomechanically superior to the RAFN with regard to construct stiffness and prevention of subsidence after cycling. This correlation between BMD and subsidence was most evident in two matched pairs with t-scores of -5.7 and 3.3 in which the two RAFN constructs after a few cycles and the LCPs failed after 68,000 and 100,000 cycles.

The present study was primarily focused on the fixation strength of each implant construct, thus the boundary conditions applied to the overall construct were of the utmost importance and any difference in the experimental constraints would affect the overall construct behavior, making direct comparison between studies difficult. The hip joint was simulated with a spherical joint in contact with the femoral head, which allows for free rotation with anatomical center of rotation. The knee joint was simulated by allowing one axis of rotation, in the flexion-extension direction. This approximated, but certainly did not exactly simulate, anatomical knee joint motion since rollback and rotation were not allowed. Nevertheless, postoperative ambulation after fixation of a distal femur fracture typically does not include deep knee flexion.

The experimental setup and test procedure in the present study were designed to simulate physiological loading during normal gait. To limit the test time to 2 days for simulating a 6-week post-operative recovery period, cyclic loading was performed at 10 
Hz. Since the change in stiffness was almost the same (7\% and 10\% for LCP and RAFN) it was assumed that the effect of loading rate on failure was the same for both constructs.

Although biomechanical studies of distal femoral fracture fixation have been reported, most studies utilized implants that are not commonly used today.(Chen et al., 2008; Heiney et al., 2009; Higgins et al., 2007; Marti et al., 2001; Sears et al., 2004; Zlowodzki et al., 2004; Zlowodzki et al., 2006) Moreover, previous studies utilized normal bone or synthetic bone despite the fact that questions and problems regarding fixation primarily occur with osteoporotic bone.(Chen et al., 2008; Heiney et al., 2009; Otto et al., 2009; Zlowodzki et al., 2006) To the knowledge of the authors, the investigated implants in this study have been previously been examined by Ito et al. (1998 and 2001), Heiney et al. (2007) and Wähnert et al. (2010) and there has not been any study investigating both implants in a matched pair comparative study.

Ito et al. tested the RAFN using seven cadaveric femur pairs and measured gap deformation in the simulated 33-A3 fracture to report the axial stiffness $(1.48 \mathrm{kN} / \mathrm{mm}$ Standard Deviation: 1.23) and load to failure (2.60 kN Standard Deviation: 0.69).(Ito et al., 1998; Ito et al., 2001) BMD was measured in the metaphyseal region as opposed to the standard hip protocol used in the present study, which makes direct comparison between BMD results difficult. The reported axial stiffness is higher but not significantly different from the results of present study $(\mathrm{p}=0.81$ and $p=0.23$ for normal and low BMD groups, respectively). Additionally, they did not find a correlation between BMD and construct stiffness or strength which is counterintuitive. 
Heiney et al. investigated the LCP using high density synthetic young bones (density was not reported) with a 33-A3 fracture and reported an axial stiffness of 625 $\mathrm{N} / \mathrm{mm}$.(Heiney et al., 2009) Only the distal femur was tested with the midshaft transected and the hip joint was omitted. The knee joint was simulated with a frictionless plate, free in lateral-medial direction, which caused excessive varus bending. These boundary conditions allowed more deformation in the fracture gap and consequently resulted in a more compliant fixation. Three LCPs were tested under cyclic loading and failure was reported in the implant after approximately 20,000 cycles without any failure in the bone, which may be due to the high level of loading (2670 N).

Wähnert et al. tested the RAFN using 8 pairs of cadaveric femurs with a 33-C2 fracture and reported $358 \pm 37 \mathrm{~N} / \mathrm{mm}$ axial stiffness and failure, defined as $2.5 \mathrm{~mm}$ subsidence, occurred after $6210 \pm 1690$ cycles.(WÄHNERT et al., 2010) The center of rotation of the simulated hip did not coincide with the anatomical hip center and there were extra degrees of freedom in the simulated knee joint, which all could explain the lower axial stiffness. A relatively large subsidence of the construct after a few thousands cycles could be due to fixing of a C2 type fracture using a RAFN without a separate lag screw, which can cause inadequate fixation in intercondylar fracture site and, as they reported, rapid loss of reduction in the intercondylar fracture gap and distal gap widening. In their study, a simple intraarticular split was created yet was treated simply with insertion of the retrograde nails. Standard principles of open reduction and internal fixation would call for lag screw compression of the fracture model used in this study (in addition to the nail or plate). 
Although physiological gait simulation was applied in the current investigation, clinical correlative studies would be needed before suggesting the safety of immediate full weight bearing of supracondylar femur fractures in osteoporotic bone with either device. Any effects of bone healing, which could clearly affect the biomechanics after fracture fixation during the six weeks of postoperative recovery, were not taken into account. The data showed that there is a correlation between bone mineral density and subsidence. Further interpretation suggests that immediate weight-bearing after either RAFN nailing with the spiral blade or locked plate fixation is safe if the surgeon is willing to accept $1 \mathrm{~mm}$ of subsidence in normal BMD and $4 \mathrm{~mm}$ in low BMD patients except in cases of severe osteoporosis. This was most evident in the two cases of catastrophic subsidence which occurred in osteoporotic specimens. The risks of this must be weighed against the risks of not allowing the patient to weight-bear as it is practiced for proximal femur fractures. Currently, the treating surgeon may not know the patient's t-score in order to predict the potential of fixation failure with early weight-bearing, however, with increasing recognition, screening, and treatment of osteoporosis, information from bone density imaging will potentially become more readily available for these patients.

In conclusion, the RAFN constructs experienced greater subsidence and reduced axial stiffness compared with the LCP in the supracondylar fracture model when subjected to post-op gait-simulation testing. If maximum $4 \mathrm{~mm}$ of subsidence is acceptable, immediate weight-bearing after either LCP or RAFN can potentially be done. However, early weight bearing is not recommended in severe osteoporotic bones treated with RAFN and may be safer with LCP. 


\section{REFERENCES}

Bolhofner, B. R., Carmen, B., Clifford, P., 1996. The results of open reduction and internal fixation of distal femur fractures using a biologic (indirect) reduction technique. Journal of Orthopaedic Trauma 10, 372.

Chen, S. H., Yu, T. C., Chang, C. H., Lu, Y. C., 2008. Biomechanical analysis of retrograde intramedullary nail fixation in distal femoral fractures. The Knee 15, 384-389.

Court-Brown, C. M., Caesar, B., 2006. Epidemiology of adult fractures: A review. Injury 37, 691-697.

Heiney, J. P., Barnett, M. D., Vrabec, G. A., Schoenfeld, A. J., Baji, A., Njus, G. O., 2009. Distal femoral fixation: a biomechanical comparison of trigen retrograde intramedullary (im) nail, dynamic condylar screw (DCS), and locking compression plate (LCP) condylar plate. The Journal of Trauma 66, 443.

Higgins, T. F., Pittman, G., Hines, J., Bachus, K. N., 2007. Biomechanical analysis of distal femur fracture fixation: fixed-angle screw-plate construct versus condylar blade plate. Journal of Orthopaedic Trauma 21, 43.

Ito, K., Grass, R., Zwipp, H., 1998. Internal fixation of supracondylar femoral fractures: comparative biomechanical performance of the 95-degree blade plate and two retrograde nails. Journal of Orthopaedic Trauma 12, 259.

Ito, K., Hungerbühler, R., Wahl, D., Grass, R., 2001. Improved intramedullary nail interlocking in osteoporotic bone. Journal of Orthopaedic Trauma 15, 192.

Marti, A., Fankhauser, C., Frenk, A., Cordey, J., Gasser, B., 2001. Biomechanical evaluation of the less invasive stabilization system for the internal fixation of distal femur fractures. Journal of Orthopaedic Trauma 15, 482.

Martinet, O., Cordey, J., Harder, Y., Maier, A., Bühler, M., Barraud, G., 2000. The epidemiology of fractures of the distal femur. Injury 31, 62-63.

Otto, R. J., Moed, B. R., Bledsoe, J. G., 2009. Biomechanical comparison of polyaxialtype locking plates and a fixed-angle locking plate for internal fixation of distal femur fractures. Journal of Orthopaedic Trauma 23, 645.

Sears, B. R., Ostrum, R. F., Litsky, A. S., 2004. A mechanical study of gap motion in cadaveric femurs using short and long supracondylar nails. Journal of Orthopaedic Trauma 18, 354.

Stover, M., 2001. Distal femoral fractures: Current treatment, results and problems* 1. Injury 32, 3-13. 
WÄHNERT, D., HOFFMEIER, K. L., VON OLDENBURG, G., FRÖBER, R., HOFMANN, G. O., MÜCKLEY, T., 2010. Internal fixation of type-C distal femoral fractures in osteoporotic bone. Journal of Bone and Joint Surgery.American Volume 92, 1442-1452.

Zlowodzki, M., Williamson, S., Cole, P., Zardiackas, L., Kregor, P., 2004.

Biomechanical evaluation of the less invasive stabilization system, angled blade plate, and retrograde intramedullary nail for the internal fixation of distal femur fractures. Unfallchirurg 107, 1107-1108.

Zlowodzki, M., Williamson, S., Zardiackas, L. D., Kregor, P. J., 2006. Biomechanical evaluation of the less invasive stabilization system and the 95-degree angled blade plate for the internal fixation of distal femur fractures in human cadaveric bones with high bone mineral density. The Journal of Trauma 60, 836. 